\title{
BMJ Open Do biological disease-modifying antirheumatic drugs reduce the spinal fracture risk related to ankylosing spondylitis? A longitudinal multiregistry matched cohort study
}

Yohan Robinson, ${ }^{1}$ Claes Olerud, ${ }^{1}$ Johan Willander ${ }^{2}$

To cite: Robinson Y, Olerud C, Willander J. Do biological disease-modifying antirheumatic drugs reduce the spinal fracture risk related to ankylosing spondylitis? A longitudinal multiregistry matched cohort study. BMJ Open 2017;7:e016548. doi:10.1136/ bmjopen-2017-016548

- Prepublication history and additional material for this paper are available online. To view these files, please visit the journal online (http://dx.doi. org/10.1136/bmjopen-2017016548).

Received 20 June 2017 Revised 1 November 2017 Accepted 14 November 2017

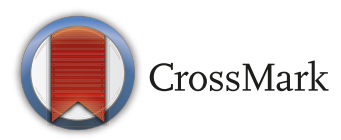

${ }^{1}$ Department of Surgical Sciences, Uppsala University Hospital, Uppsala, Sweden ${ }^{2}$ Department of Psychology, Gävle University College, Gävle, Sweden

Correspondence to Dr Yohan Robinson; yohan.robinson@surgsci.uu.se

\section{ABSTRACT}

Objectives Ankylosing spondylitis (AS) is associated with an increased spinal fracture risk due to the loss of elasticity in spinal motion segments. With the introduction of biological disease-modifying antirheumatic drug (bDMARD) treatment for AS, the individual course of the disease has been ameliorated. This study aims to examine the association of bDMARD treatment and risk of spinal fracture.

Design Longitudinal population-based multiregistry observational matched cohort study.

Setting Swedish Patient Registry 1987-2014 and Swedish Prescribed Drugs Registry 2005-2014.

Participants Included were patients $\geq 18$ years of age receiving treatment at a healthcare facility for the primary diagnosis of AS. About 1352 patients received more than one prescription of bDMARD from 2005 to 2014. An untreated control group was created by propensity score matching for age, sex, comorbidity, antirheumatic prescriptions and years with AS $(n=1352)$.

Main outcome measures Spinal fracture-free survival. Results No bDMARD treatment-related effect on spinal fracture-free survival was observed in the matched cohorts. Male gender ( $\mathrm{HR}=2.54,95 \% \mathrm{Cl} 1.48$ to 4.36 ) and Charlson Comorbidity Index score $(\mathrm{HR}=3.02,95 \% \mathrm{Cl} 1.59$ to 5.75) contributed significantly to spinal fracture risk. Conclusion bDMARD had no medium-term effect on the spinal fracture-free survival in patients with AS. Trial registration number NCT02840695; Post-results.

\section{INTRODUCTION}

Ankylosing spondylitis (AS) is a rheumatic disease affecting the axial skeleton, leading to a progressive ankylosis of all spinal segments. ${ }^{1}$ Even though not all patients fuse, there is consensus on the end-stage of axial AS, the bamboo spine, being a pathognomonic radiological feature for this disease. ${ }^{2}$ Ankylosis often leads to a rigid kyphotic sagittal profile, where flexibility and segmental lever arm length are comparable to a long bone of the lower extremities.

\section{Strengths and limitations of this study}

- The national multiregistry approach provides nationwide coverage of prescriptions since 2005 and ankylosing spondylitis (AS) and spinal fracture incidence since 1987.

- With propensity score matching of treatment and control groups, the bias of the confounders year of birth, sex, comorbidity, years with AS diagnosis and comedication was addressed.

- Drugs administrated to inpatients or specialised hospital-bound clinics are not registered in the Prescribed Drugs Registry, where only outpatient prescriptions are registered.

- Since a long-term effect of antirheumatic treatment is to be assumed, a longer observation period of this cohort could change our study results.

Due to the unfavourable biomechanics, risk of falls and AS-related osteoporosis, the spine in $\mathrm{AS}$ is prone to fractures even in minor trauma. ${ }^{3}$ Spinal injury prevention of patients with an ankylosed spine nowadays comprises of improvement of balance and posture with physiotherapy and rehabilitation. While restriction of certain activities can reduce the risk of spinal fractures in end-stage AS, the proven benefit of training on pulmonary function and quality of life often outweighs these risks. ${ }^{4}$

Currently, there is no evidence for the efficacy of glucocorticoids, sulfasalazine and methotrexate for the treatment of axial AS. ${ }^{5}$ Non-steroidal anti-inflammatory drugs (NSAID) are recommended as first-line drug treatment for patients with AS with pain and stiffness. For patients with persistently high disease activity despite conventional treatments biological disease-modifying antirheumatic drugs (bDMARD) therapy is recommended. 
The bDMARD group consist mainly of anti-tumour necrosis factor- $\alpha$ pharmaceuticals which all have proven effect with regard to pain, function, quality of life and inflammation compared with placebo. ${ }^{6} 7$ Recently, interleukin-17A inhibitors have been added to the bDMARD family for the treatment of AS. ${ }^{8}$

The long-term delay of spinal ankylosis and the risk reduction for spinal fractures has not been the primary goal of pharmacological therapy for AS, but several authors indicate that the bDMARD treatment has the potential of reducing or delaying spinal ankylosis. ${ }^{2}{ }^{9} \mathrm{~A}$ radiographical study finds reduced progression of spinal ankylosis if patients receive bDMARD for more than 4 years. ${ }^{10}$ Furthermore, bDMARD increase bone density which is of great importance regarding the spinal fracture risk. ${ }^{11}{ }^{12}$ Given the beneficial effects of bDMARD treatment, a reduced spinal fracture rate could be anticipated in patients receiving modern antirheumatic therapy. ${ }^{13}$

This study is designed to investigate whether bDMARD treatment reduces the spinal fracture risk related to AS in national registry-based observational matched cohorts.

\section{METHODS}

\section{Study design}

This national multiregistry matched cohort study uses prospectively collected electronic healthcare data from the Swedish National Patient Registry (NPR), the Swedish Cause of Death Registry (CDR) and the Swedish Prescribed Drugs Registry (PDR) between 2005 and 2014. This study protocol follows Strengthening the Reporting of Observational Studies in Epidemiology and REporting of studies Conducted using Observational Routinely collected health Data statements. ${ }^{1415}$

\section{Setting}

The Swedish NPR is hosted by the Swedish National Board of Health and Welfare and contains all patient contacts within Sweden with a coverage of $>90 \%$ for orthopaedic diagnoses. ${ }^{16}$ Registered are main diagnosis and comorbidity using International Classification of Diseases, Ninth Revision (ICD-9) until December 1996, and since then the International Classification of Diseases, 10th Revision (ICD-10) code. ${ }^{17}$ Treatment is coded since 1997 using the Swedish classification of surgical procedures. ${ }^{18}$ Furthermore, information on hospitalisation time is available from the registry. Since 2005, even outpatient/ primary care contacts were included in the NPR. ${ }^{19}$

Data collection for the Swedish PDR is administered by the National Corporation of Swedish Pharmacies, a governmental institution responsible for the provision of pharmaceutical services in the whole country. Since July 1,2005 , information from all prescriptions dispensed is monthly transferred to the Centre for Epidemiology at the National Board of Health and Welfare, responsible for keeping the registry. ${ }^{20}$ The PDR uses Anatomical Therapeutic Chemical (ATC) codes for identification of medication group.
In the Swedish CDR, all incident deaths and cause of death are registered for all patients. A validation of death certificates and CDR registration document found $83 \%$ agreement for hospital and $46 \%$ agreement for nonhospital cause of death. ${ }^{21}$

\section{Participants}

After removal of 18551 duplicate entries, the original registry extract from the NPR contained 13112 patients registered with the main diagnosis of AS treated between 1 January 1987 and 31 December 2014. A second dataset was provided from the PDR including 1474949 prescriptions of anti-inflammatory drugs to patients in the dataset from the NPR between 1 July 2005 and 31 December 2014. Prior to data transmission, the Swedish National Board of Health and Welfare anonymised the individual personal identification numbers using a key which remained with the agency.

\section{Variables}

The ICD-9 code ' 720 ' and the ICD-10 code 'M45' were used to identify patients with AS in the NPR. From the NPR, baseline values such as age, gender and date of hospitalisation were collected for each included individual. Additionally, comorbidity was collected by storing coincident ICD-9 and ICD-10 codes in each patient's entry. Using ICD-codes, the Charlson Comorbidity Index (CCI) could be calculated for each patient using a previously validated algorithm. ${ }^{22}$ The factor 'CCI score' categorised the level of comorbidity in low (1-3), moderate (4-5), high (6-7) and very high comorbidity $(\geq 8)$.

Patients with spinal fractures from 2005 to 2014 were identified in the NPR using ICD-10 codes S12.0, S12.1, S12.2, S12.7, S12.8, S12.9, S13.0, S13.1, S13.2, S13.3, S22.0, S22.1, S23.0, S23.1, S32.0, S32.1, S33.0 and S33.1. Even ICD-10 codes for osteoporotic spinal fractures M80.0A, $\mathrm{M} 80.0 \mathrm{~J}$ and $\mathrm{M} 80.0 \mathrm{~K}$, and delayed/non-unions M84.0J, M84.0K, M84.1A, M84.1J, M84.1K, M84.2A, M84.2J and M84.2K were included. Patients with a history of rheumatoid arthritis (ICD-9: 714, ICD-10: M05 and M06) were excluded from the analysis, as they could have received bDMARD treatment for different reasons.

All dates of death of included patients were extracted from the CDR. This allowed censoring of the fracture-free survival analysis using the true dates of death.

To identify anti-inflammatory prescriptions, the PDR was searched for the ATC codes for bDMARD (L04AA and L04AB), NSAID (M01A), methotrexate (L04AX) and sulfasalazine (A07EC01). Registered were number of prescriptions and years of treatment.

\section{Statistical methods}

All statistical calculations were programmed in R V.3.3.0 (R Foundation for Statistical Computing, Vienna, Austria) ${ }^{23}$ Patients receiving bDMARD were matched against an untreated control group using the 'MatchIT' package. Propensity score matching was performed 1:1 for year of birth, sex, CCI and years with the diagnosis of 


\section{CONSORT 2010 Flow Diagram}

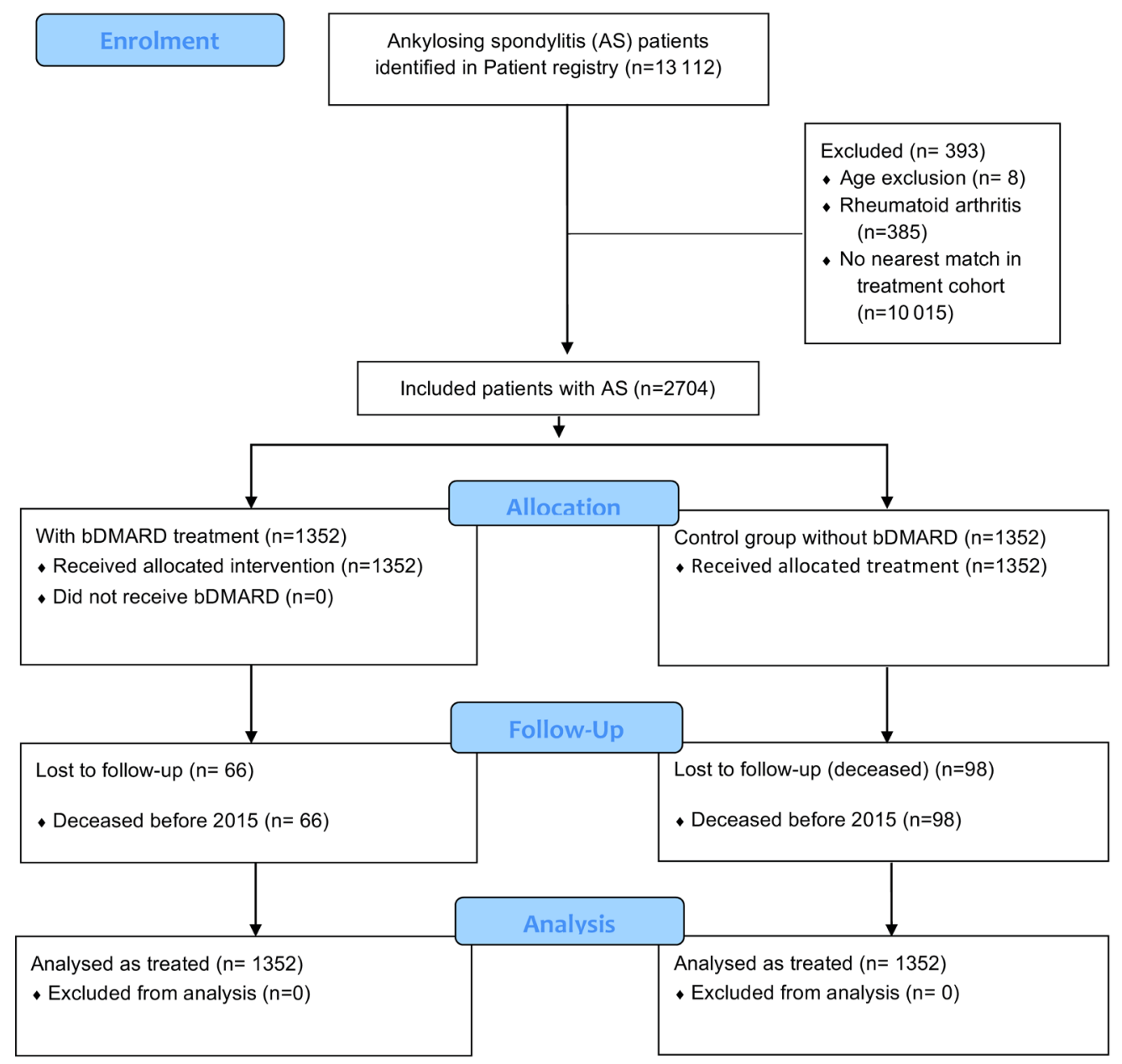

Figure 1 CONSORT inclusion flow diagram. bDMARD, biological disease-modifying antirheumatic drugs; CONSORT, Consolidated Standards of Reporting Trials.

AS, with the hierarchical 'nearest' neighbour matching method. Online supplementary appendix 1 summarised the propensity scores of treatment, matched control and unmatched control groups. The included participants were grouped according to bDMARD treatment in bDMARD and (untreated) control groups. An inclusion flow diagram according to Consolidated Standards of Reporting Trials (CONSORT) statements illustrated the inclusion protocol. ${ }^{24}$

As relevant covariates for the occurrence of a spinal fracture, besides years of bDMARD treatment, years of NSAID treatment ${ }^{325}$ and male gender ${ }^{26}$ were identified from systematic literature review. The effect of bDMARD on spinal fracture-free survival in the matched cohorts was estimated using a Cox proportional hazards model applying the right censored type of the 'Surv()' function in the 'survival' package in R. Both univariate and multivariate models were developed. Fracture-free survival was visualised using a Kaplan-Meier plot adjusted for gender, CCI score and years of bDMARD treatment.

The values are expressed as mean $\pm \mathrm{SD}$, if not indicated otherwise. Groups were compared with t-test for normally distributed variables, otherwise the Wilcoxon test was applied. Group proportions were tested with the $\chi^{2}$ test. A probability of $\mathrm{P}<0.05$ was considered as statistically significant.

\section{Data access and cleaning methods}

The authors did not have direct access to the national registry databases in this study, but were provided a predefined extract from the national registries by the 
Table 1 Baseline data of matched cohorts with and without bDMARD treatment with $P$ values of group differences

\begin{tabular}{llllr}
\hline & & bDMARD & Control & P value \\
\hline $\mathrm{n}$ & & 1352 & 1352 & \\
Age & Years & $55 \pm 12$ & $53 \pm 12$ & $<0.001$ \\
Female proportion & & $36 \%$ & $36 \%$ & 0.689 \\
$\mathrm{CCl}$ & & $2.8 \pm 1.8$ & $2.6 \pm 1.8$ & 0.008 \\
$\begin{array}{l}\text { Time since AS } \\
\text { diagnosis }\end{array}$ & Years & $12 \pm 8$ & $12 \pm 8$ & 0.831 \\
Methotrexate & Years & $6 \pm 18$ & $4 \pm 11$ & $<0.001$ \\
Sulfasalazine & Years & $4 \pm 13$ & $4 \pm 13$ & 0.409 \\
NSAID & Years & $20 \pm 19$ & $20 \pm 24$ & 0.833 \\
\hline
\end{tabular}

AS, ankylosing spondylitis; bDMARD, biological disease-modifying antirheumatic drugs; $\mathrm{CCI}$, Charlson Comorbidity Index; NSAID, non-steroidal anti-inflammatory drugs.

Swedish National Board of Health and Welfare (specification no: 13062/2015).

Even though a clean patient registry dataset was provided, duplicates (recurrent admissions of the same patient or continued treatment in a secondary facility) had to be identified and removed from the extract. Prior to removal of duplicates, comorbidity data from duplicate records were stored in the unique patient record.

From the PDR for each included patient, the number of prescriptions from 2005 to 2014 was extracted, as well as the number of years from 2005 to 2014 when the patient received antirheumatic treatment.

\section{Linkage}

Individual patients in all three registries were identifiable by unique identification numbers. By searching the patient registry for diagnosis of AS, a duplicate-free dataset of all included patients was created. The CDR was linked with this dataset using the 'merge()' function in R. For each patient in the dataset, the number and years of prescriptions were identified after splitting the PDR according to identification number, and then searching for prescriptions. Due to the unique identification number used in all three registries, the linkage quality which was controlled with 50 random samples was $100 \%$.

\section{RESULTS}

Participants

All 1352 included patients with AS receiving bDMARD were matched with 1352 controls with AS without bDMARD treatment. The CONSORT inclusion flow diagram is depicted in figure 1.

\section{Descriptive data}

All 1352 patients in the treatment group received bDMARD treatment (6166 person-years of treatment) and were followed for median 10 years after AS diagnosis (16567 person-years of observation), while the 
Table 2 Fracture-free survival of patients with and without bDMARD treatment

\begin{tabular}{|c|c|c|c|c|c|c|c|}
\hline \multirow[b]{2}{*}{ Covariate } & \multirow[b]{2}{*}{ Categories } & \multirow{2}{*}{$\begin{array}{l}\text { Fractures/ } \\
\text { subjects, } n\end{array}$} & \multirow{2}{*}{$\begin{array}{l}\text { Person- } \\
\text { years, } n \\
\text { bDMARD }\end{array}$} & \multicolumn{2}{|l|}{ Univariate } & \multicolumn{2}{|c|}{ Multivariate* } \\
\hline & & & & HR $(95 \% \mathrm{Cl})$ & $P$ value & HR (95\% Cl) & $P$ value \\
\hline \multicolumn{8}{|l|}{ Treatment } \\
\hline & Control & $44 / 1352$ & 17 & 1.00 (Ref) & & 1.00 (Ref) & \\
\hline & bDMARD & $47 / 1352$ & 6166 & 1.05 (0.70 to 1.59$)$ & 0.804 & $1.00(0.66$ to 1.51$)$ & 0.999 \\
\hline \multicolumn{8}{|l|}{ Gender } \\
\hline & Female & $16 / 973$ & 2050 & 1.00 (Ref) & & 1.00 (Ref) & \\
\hline & Male & $75 / 1731$ & 4133 & 2.54 (1.48 to 4.36$)$ & $<0.001$ & 2.40 (1.40 to 4.13$)$ & 0.002 \\
\hline \multicolumn{8}{|l|}{$\mathrm{CCl}$ score } \\
\hline & Low (1-3) & $51 / 1981$ & 4481 & 1.00 (Ref) & & 1.00 (Ref) & \\
\hline & Moderate (4-5) & $28 / 585$ & 1430 & 1.68 (1.05 to 2.66$)$ & 0.028 & 1.61 (1.02 to 2.56$)$ & 0.043 \\
\hline & High (6-7) & $7 / 94$ & 194 & 2.70 (1.22 to 5.95$)$ & 0.014 & 2.55 (1.16 to 5.63$)$ & 0.020 \\
\hline & Very high $(\geq 8)$ & $5 / 44$ & 78 & 4.44 (1.77 to 11.12$)$ & 0.001 & 3.91 (1.55 to 9.82$)$ & 0.004 \\
\hline
\end{tabular}

Results of univariate and multivariate Cox proportional hazards regression are presented with $95 \% \mathrm{Cl}$.

${ }^{*} r^{2}=0.009$ (likelihood ratio test $P<0.001$, Wald test $P<0.001$, score (log-rank) test $P<0.001$ ).

bDMARD, biological disease-modifying antirheumatic drugs; CCI, Charlson Comorbidity Index; Ref, reference category.

1352 in the control group received no bDMARD treatment more than once (16 person-years of treatment) and were followed for median 8 years (16189 person-years). Those receiving bDMARD were older $(\mathrm{P}<0.001)$, had a higher CCI $(\mathrm{P}=0.008)$ and received more methotrexate $(\mathrm{P}<0.001)$ than the control group. Of 2704 patients with AS, 91 had a fracture. The baseline data of the matched cohorts are presented in table 1 .

\section{Main results}

Patients with bDMARD had a spinal fracture after 12 years with the registered diagnosis AS (95\% CI 6 to 12), and those without after 11 years (95\% CI 5 to 12). Regarding fracture-free survival, no bDMARD treatment effect was observed, neither in the univariate model ( $\mathrm{HR}=1.05,95 \%$ CI 0.70 to $1.59, \mathrm{P}=0.80$ ), nor in the multivariate model ( $\mathrm{HR}=1.00,95 \%$ CI 0.66 to $1.51, \mathrm{P}=0.99)$. Instead, male gender $(\mathrm{HR}=2.54,95 \%$ CI 1.48 to $4.36, \mathrm{P} \leq 0.001)$ and CCI score $(\mathrm{HR}=3.02,95 \%$ CI 1.59 to $5.75, \mathrm{P}<0.001)$ contributed significantly to fracture risk. Adjusted survival curves by treatment are presented in figure 2 . Table 2 summarises the results from univariate and multivariate Cox proportional hazards regression models.

\section{DISCUSSION}

\section{Principal findings}

This study analysed in a national multiregistry cohort the spinal fracture risk of all patients with AS treated with bDMARD. No effect of bDMARD treatment on the spinal fracture risk related to AS was found.

\section{Strengths and weaknesses of this study}

Even though registry studies have obvious advantages providing nationwide population data, several limitations and sources of bias must be assumed.
One important limitation of this study is the relatively short observation time for the medication data, which are only available since 2005. Since a long-term effect of antirheumatic treatment is to be assumed, longer observation periods of this cohort could change the presented results. We strongly recommend revisiting this cohort in the future to audit our assumptions based on medium-term follow-up.

A major observational error is implicit with the design of the PDR, registering only prescribed drugs picked up at pharmacies. ${ }^{20}$ Drugs administrated to inpatients or specialised hospital-bound clinics are not registered in the PDR, implying a significant source of bias. ${ }^{27}$

Another limitation of this study is the fact that a prescription and expenditure registered in the PDR does not mean that the patient took his medication. There is abundant data that patients only take about $50 \%$ of their prescribed medication. ${ }^{28}$ With regard to our study, the actual treatment effect could have been reduced due to this bias.

One of the peculiarities with AS is the inflammatory interval between the late $20 \mathrm{~s}$ and the $60 \mathrm{~s}$, followed by a rheumatologically asymptomatic but structurally vulnerable phase where an ankylosed and osteoporotic axial skeleton remains with a high-fracture risk. ${ }^{29}$

Regional differences in the accessibility of healthcare in Sweden could delay the primary diagnosis of AS. These data were not included in the registry extract of our study and could not be adjusted for.

The validation of the NPR using other quality registries confirms high validity of registered orthopaedic diagnoses. ${ }^{16}$ Diagnoses as hip fractures are correctly identified in more than $95 \%$. Since the NPR was started in the 1960s, a coding learning curve could explain an increasing incidence for most diagnosis groups. Instead, no increasing 
incidence of lumbar fractures was reported in the NPR during the last decades, which would have been expected if a systematic bias was present. ${ }^{30}$ Obviously, the current registration quality is good, and registration bias cannot explain the findings in this study. Besides, the Swedish reimbursement policy requires complete diagnosis registration, an effective incitement to proper coding.

This study presents data from the geographically, health-economically and ethnically unique country Sweden, which cannot be generalised to other countries. Future studies from national prescription registries in other countries will have to validate our results in their unique setting.

As with most therapeutic registry studies, our results are prone to the selection of an inadequate reference group. It is very likely that those receiving bDMARD have greater access to high quality healthcare and were possibly screened for AS at an earlier age, thus receiving adequate physiotherapy and prevention, while those with less access were possibly diagnosed with AS together with their first spinal fracture when bDMARD treatment is not an option anymore. ${ }^{31}$ In contrast, it would even be possible that patients with bDMARD treatment have a more therapy resistant form of AS and thus receive this still expensive treatment. Those without bDMARD were then relatively symptom free with NSAID treatment. Thus, bDMARD treatment could be associated with a more aggressive course of the disease, biasing the bDMARD group towards worse results. We addressed this bias by matching the control group even for years with AS diagnosis.

\section{Strengths and weaknesses in relation to other studies}

One of the most recognised complications of AS are spinal fractures, which are associated with multiple potentially hazardous complications. ${ }^{3}$ Reason for the susceptibility of the ankylosed spine to fractures are long lever arms of the stiff spine, ${ }^{32}$ as well as osteoporosis related to this rheumatic disease. ${ }^{33}$

Interestingly, other authors found an effect of antirheumatic drugs on spinal fracture incidence related to AS. Muñoz-Ortego et $a \hat{l}^{\beta}$ presented Spanish national population-based registry data from 2006 in 6474 patients with AS, suggesting regular NSAID treatment to reduce spinal fracture risk $(\mathrm{P}=0.02)$. Unfortunately, they did not include bDMARD treatment in their analysis. Vosse $e t a l^{25}$ made similar conclusions from a UK General Practice Research Database extract from 1988 to 1999, where NSAID but not sulfasalazine was associated with a reduced spinal fracture rate related to AS (OR 0.65; 95\% CI 0.50 to 0.84 ). Here, again bDMARD were not investigated, most likely due to the historical data, when bDMARD treatment was not common practice.

In a prospectively collected cohort of 173 patients with AS treated with bDMARD, Maas $e t a l^{34}$ found that during 4 years of bDMARD therapy, 20\% developed at least one new radiographic vertebral fracture. They found that older age, smoking and osteoporosis at baseline to be associated with the development of new spinal fractures.

\section{Meaning of the study}

This study suggests that bDMARD has no medium-term effect on the risk of spinal fractures related to AS. Therefore, recommendations for physiotherapeutic guidance for spinal injury prevention are valid even for patients receiving bDMARD. ${ }^{35}$

\section{Unanswered questions and future research}

The follow-up of 10 years may have underestimated both beneficial and adverse effects of bDMARD. Therefore, studies revisiting this unique national cohort are recommended to validate the findings in this study. Beyond that, long-term follow-up of available cohorts from randomised placebo-controlled trials on bDMARD treatment ${ }^{6}$ could provide important post hoc data regarding the treatment effect on spinal fracture incidence and its health economic implications.

Contributors YR: designed the study, analysed the data and wrote the manuscript. $\mathrm{CO}$ : supervised the study and revised the final manuscript. JW: critically revised the statistical methods.

Competing interests YR and $\mathrm{CO}$ have been paid for developing and delivering educational presentations for Medtronic and DePuy Synthes.

Ethics approval Uppsala Ethical Review Board (no: 2015/147).

Provenance and peer review Not commissioned; externally peer reviewed.

Data sharing statement The Swedish National Patient Registry is hosted by the Swedish National Board of Health and Welfare. Data collection for the Swedish Prescribed Drugs Registry (PDR) is administered by the National Corporation of Swedish Pharmacies. National registry data is available to researchers from the Swedish National Board of Health and Welfare (http://www.socialstyrelsen.se/ statistics) after institutional review board approval.

Open Access This is an Open Access article distributed in accordance with the Creative Commons Attribution Non Commercial (CC BY-NC 4.0) license, which permits others to distribute, remix, adapt, build upon this work non-commercially, and license their derivative works on different terms, provided the original work is properly cited and the use is non-commercial. See: http://creativecommons.org/ licenses/by-nc/4.0/

(c) Article author(s) (or their employer(s) unless otherwise stated in the text of the article) 2017. All rights reserved. No commercial use is permitted unless otherwise expressly granted.

\section{REFERENCES}

1. Boonen A, Brinkhuizen T, Landewé R, et al. Impact of ankylosing spondylitis on sick leave, presenteeism and unpaid productivity, and estimation of the societal cost. Ann Rheum Dis 2010;69:1123-8.

2. Haroon N, Danda D. 'Bamboo spine', a thing of the past? Int J Rheum Dis 2014;17:127-30.

3. Muñoz-Ortego J, Vestergaard P, Rubio JB, et al. Ankylosing spondylitis is associated with an increased risk of vertebral and nonvertebral clinical fractures: a population-based cohort study. J Bone Miner Res 2014;29:1770-6.

4. Baraliakos X, Sewerin P. Sport mit morbus bechterew? Warum? Und wenn ja: wie? Morbus-Bechterew Journal 2017;2017:18-20.

5. Braun J, van den Berg R, Baraliakos X, et al. 2010 update of the ASAS/EULAR recommendations for the management of ankylosing spondylitis. Ann Rheum Dis 2011;70:896-904.

6. Maxwell LJ, Zochling J, Boonen A, et al. TNF-alpha inhibitors for ankylosing spondylitis. Cochrane Database Syst Rev 2015;4:Cd005468.

7. Prince DS, McGuigan LE, McGirr EE. Working life and physical activity in ankylosing spondylitis pre and post anti-tumor necrosis factor-alpha therapy. Int J Rheum Dis 2014;17:165-72.

8. Baeten D, Sieper J, Braun J, et al. Secukinumab, an Interleukin-17A Inhibitor, in Ankylosing Spondylitis. N Engl J Med 2015;373:2534-48.

9. Braun J, Baraliakos X, Deodhar A, et al. Effect of secukinumab on clinical and radiographic outcomes in ankylosing spondylitis: 2-year 
results from the randomised phase III MEASURE 1 study. Ann Rheum Dis 2017;76:1070-7.

10. Maas F, Arends S, Brouwer E, et al. Reduction in spinal radiographic progression in ankylosing spondylitis patients receiving prolonged treatment with TNF-alpha inhibitors. Arthritis Care Res 2016. doi: 10.1002/acr.23097. [Epub ahead of print 4 Oct 2016].

11. Haroon NN, Sriganthan J, Al Ghanim N, et al. Effect of TNFalpha inhibitor treatment on bone mineral density in patients with ankylosing spondylitis: a systematic review and meta-analysis. Semin Arthritis Rheum 2014;44:155-61.

12. Briot $\mathrm{K}$, Roux $\mathrm{C}$. Inflammation, bone loss and fracture risk in spondyloarthritis. RMD Open 2015;1:e000052.

13. Robinson Y, Willander J, Olerud C. Surgical stabilisation improves survival of spinal fractures related to ankylosing spondylitis. Spine 2016. [Epub ahead of print 11 Aug 2015]

14. Benchimol El, Smeeth L, Guttmann A, et al. The REporting of studies Conducted using Observational Routinely-collected health Data (RECORD) statement. PLoS Med 2015;12:e1001885.

15. von Elm E, Altman DG, Egger M, et al. The Strengthening the Reporting of Observational Studies in Epidemiology (STROBE) statement: guidelines for reporting observational studies. Int J Surg 2014;12:1495-9.

16. Ludvigsson JF, Andersson E, Ekbom A, et al. External review and validation of the Swedish national inpatient register. BMC Public Health 2011:11:450.

17. World Health Organisation. International statistical classification of diseases and related health problems. 2006. http://www3.who.int/ icd/currentversion/fr-icd.htm2010

18. Socialstyrelsen. In: Grafiska B, ed. Classification of surgical procedures version 1.9. Revised version of 2004. Lindesberg, Sweden, 2004.

19. Emilsson L, Lindahl B, Köster M, et al. Review of 103 swedish healthcare quality registries. J Intern Med 2015;277:94-136.

20. Wettermark B, Hammar N, Fored CM, et al. The new swedish prescribed drug register-opportunities for pharmacoepidemiological research and experience from the first six months. Pharmacoepidemiol Drug Saf 2007:16:726-35.

21. Johansson LA, Westerling R. Comparing Swedish hospital discharge records with death certificates: implications for mortality statistics. Int J Epidemiol 2000;29:495-502.
22. Sundararajan V, Henderson T, Perry C, et al. New ICD-10 version of the Charlson comorbidity index predicted in-hospital mortality. J Clin Epidemiol 2004;57:1288-94.

23. R Core Team. R: a language and environment for statistical computing. Vienna, Austria, 2017.

24. Moher D, Hopewell S, Schulz KF, et al. CONSORT 2010 explanation and elaboration: updated guidelines for reporting parallel group randomised trials. BMJ 2010;340:c869.

25. Vosse D, Landewé R, van der Heijde D, et al. Ankylosing spondylitis and the risk of fracture: results from a large primary care-based nested case-control study. Ann Rheum Dis 2009;68:1839-42.

26. Caron T, Bransford R, Nguyen Q, et al. Spine fractures in patients with ankylosing spinal disorders. Spine 2010;35:E458-E464.

27. Linder M, Byström C, Kieler $\mathrm{H}$, et al. Use of palivizumab is underestimated in the Swedish Prescribed Drug Register implications for register-based drug studies. Clin Epidemiol 2015;7:45-51.

28. Brown MT, Bussell JK. Medication adherence: WHO cares? Mayo Clin Proc 2011;86:304-14.

29. Lee W, Reveille JD, Davis JC, et al. Are there gender differences in severity of ankylosing spondylitis? Results from the PSOAS cohort. Ann Rheum Dis 2007;66:633-8.

30. Robinson Y, Sandén B, Olerud C. Increased occurrence of spinal fractures related to ankylosing spondylitis: a prospective 22-year cohort study in 17,764 patients from a national registry in Sweden. Patient Saf Surg 2013;7:2.

31. Kalkan A, Husberg M, Hallert E, et al. Physician preferences and variations in prescription of biologic drugs for rheumatoid arthritis: $A$ register-based study of 4,010 patients in Sweden. Arthritis Care Res 2015;67:1679-85.

32. Robinson Y, Robinson AL, Olerud C. Complications and survival after long posterior instrumentation of cervical and cervicothoracic fractures related to ankylosing spondylitis or diffuse idiopathic skeletal hyperostosis. Spine 2015;40:E227-E233.

33. Carter S, Lories RJ. Osteoporosis: a paradox in ankylosing spondylitis. Curr Osteoporos Rep 2011;9:112-5.

34. Maas F, Spoorenberg A, Brouwer E, et al. Radiographic vertebral fractures develop in patients with ankylosing spondylitis during 4 years of TNF- $\alpha$ blocking therapy. Clin Exp Rheumatol 2016;34:191-9.

35. Millner JR, Barron JS, Beinke KM, et al. Exercise for ankylosing spondylitis: An evidence-based consensus statement. Semin Arthritis Rheum 2016;45:411-27. 